\title{
Hemicraniectomy after massive hemispheric cerebral infarction: are we ready for a prospective randomised controlled trial?
}

\section{K M Cockroft}

\section{Quality of survival after hemicraniectomy remains a key factor}

$\mathrm{H}$ emicraniectomy for massive cerebral infarction remains a controversial issue within neurosurgery and neurology. In this issue, Uhl et $a l^{1}$ provide us with valuable insight into the factors that influence outcome in this situation. Their multi-institution report is the largest case series of patients who have undergone decompressive craniectomy for massive space occupying cerebral infarction. The pooling of databases from eight neurosurgical departments resulted in the inclusion of 188 patients with a median follow up of 26 weeks. The authors assessed various prognostic factors including sex, age, presence of anisocoria, Glasgow coma score, extension of infarction, laterality, pre-existing medical conditions, and time from infarction to surgery. Unadjusted three, six, and 12 month mortality rates were $7.9 \%, 37.6 \%$, and $43.8 \%$, respectively. In the multivariate analysis, only age (over 50 years) and extension of infarction (involvement of two or more vascular territories) proved to be independent predictors of death.

Although this article suggests answers to several questions regarding the management of patients with massive hemispheric infarction, its very nature as an uncontrolled retrospective account highlights the many important questions that remain unansweredand cannot be without a prospective randomised controlled trial. Certainly, the hurdles involved in the design and implementation of such a trial are considerable. Of primary importance is the question of which patients should be enrolled and during what time period they should be randomised. On the subject of timing, Uhl et al suggest that the interval from infarction to surgery may not be particularly important for survival. This seems to go against common wisdom and previous reports. $^{23}$ However, Uhl's use of 39 hours to separate early and late procedures, in comparison with 24 hours or less used by other investigators, makes this conclusion less compelling. As to the question of whom to randomise, the answer may be even more difficult to find. Many investigators, ${ }^{4-6}$ including Uhl and his colleagues, have suggested that younger patients are likely to derive more benefit from decompressive craniectomy, both in terms of survival and functional recovery. Does this mean that young patients should not be randomised? Alternatively, does this mean that young patients should be randomised because they are the group most likely to benefit and therefore most likely to provide an outcome in favour of surgery in a randomised trial? The absence of a medically treated control group in this and other studies leaves the answer uncertain. However, it is well known that young patients tend to have a better long term outcome after ischaemic stroke in general, and given the acknowledged higher than expected mortality reported by Uhl, it may well be that the 12 month mortality rates for surgically and medically treated patients in this situation are not statistically different, suggesting that clinical equipoise still exists even among younger patients.

The choice of end points for a prospective randomised controlled trial will also be important. Not surprisingly, in Uhl's report the side of infarction did not affect survival. However, "survival" alone is probably not the most important end point in this situation. The main reason many neurosurgeons do not undertake decompressive craniectomy on patients with large dominant hemisphere infarcts is not a concern over survival, but rather a concern over the quality of that survival. While Uhl and colleagues make some effort to address functional outcome using the Glasgow outcome scale (GOS) and the
Barthel index, the GOS is a rather crude tool in this situation and the Barthel index was not available in all the surviving patients. For a prospective randomised controlled trial to provide meaningful data, a more detailed assessment of functional status and patient/primary caregiver satisfaction will need to be obtained.

In the end the prospective randomised controlled trial remains the gold standard in clinical research. At this point, there are enough data from case series such as that of Uhl et al to frame an appropriate design for such a trial involving decompressive craniectomy after massive hemispheric infarction. An age inclusive design based on early surgical intervention according to computed tomographic or magnetic resonance imaging criteria will facilitate recruitment and assist in answering the question of whom and when to treat. An intention to treat analysis that allows for crossover will help ensure a true approximation of clinical practice while at the same time serving to safeguard patients whom clinicians feel may require decompression in a delayed manner. Fortunately, at least two prospective trials are already under way. ${ }^{7}$ However, for patient recruitment in these studies to be successful, neurosurgeons and neurologists will have to temporarily put aside their existing biases with regard to the role of decompressive craniectomy in favour of enrolment in a randomised prospective trial. In exchange for the uncertainty of randomisation, there is the hope for some definitive "answers" in the future. Until the results of a prospective randomised controlled trial become available, clinicians will have to continue to rely on the case by case assessment of patients based on incomplete prognostic criteria from class II evidence.

J Neurol Neurosurg Psychiatry 2004;75:179-180.

doi: 10.1136/jnnp.2003.028407

Correspondence to: Dr Kevin M Cockroft, PO Box 850, Department of Neurological Surgery, Penn State Hershey Medical Center, Hershey,

Pennsylvania, USA; kcockroft@psu.edu

\section{REFERENCES}

1 Uhl E, Kreth F, Elias B, et al. Outcome and prognostic factors of hemicraniectomy for space occupying cerebral infarction. J Neurol Neurosurg Psychiatry 2004;75:270-4.

2 Schwab S, Steiner T, Aschoff A, et al. Early hemicraniectomy in patients with complete middle cerebral artery infarction. Stroke 1998;29: 1888-93.

3 Cho DY, Chen TC, Lee HC. Ultra-early decompressive craniectomy for malignant middle cerebral artery infarction. Surg Neurol 2003;60:227-32.

4 Walz B, Zimmerman C, Bottger S, et al. Prognosis of patients after hemicraniectomy in malignant middle cerebral artery infarction. J Neurol 2002;249: 1183-90. 
5 Carter BS, Ogilvy CS, Candia GJ, et al. One-year outcome after decompressive surgery for massive nondominant hemisphere infarction. Neurosurgery 1997;40:1 168-75.
6 Pranesh MB, Dinesh Nayak S, Mathew V, et al. Hemicraniectomy for large middle cerebral artery territory infarction: outcome in 19 patients. J Neurol Neurosurg Psychiatry 2003;74:800-2
7 Morley NCD, Berge E, Cruz-Flores S, et al. Surgical decompression for cerebral oedema in acute ischaemic stroke (Cochrane review). In: The Cochrane Library. 2003, Issue 3. Oxford: Update Software, 2003

\section{NEUROLOGICAL PICTURE}

\section{Visualisation of the circumventricular organs by fluorescence endoscopy}

$\mathrm{T}$ he circumventricular organs are highly specialised sites in the brain. They lie outside of the blood-brain barrier, are located periventricularly, and are thought to play an important role as the gateway through which information reciprocates between the outside and inside of the brain. ${ }^{1} \mathrm{We}$ have attempted to visualise the circumventricular organs with the use of a fluorescence endoscope at the time of ventriculoperitoneal shunt revision.

A 71 year old man presented with progressive decline in mental acuity and gait disturbance. A ventriculoperitoneal shunt had been placed for normal pressure hydrocephalus following cerebral contusion 20 years before. The shunt was found to be obstructed, and was replaced with a pressure programmable valve during ventriculoperitoneal shunt revision surgery. The existing ventricular catheter was extracted from the left lateral ventricle after we confirmed that there was no adhesion to the choroid plexus with the fiberscope inserted into the right lateral ventricle by observing through the defect in the septum pellucidum. We used the fiberscope, measuring $5 \mathrm{~mm}$ in diameter, to inspect the interior of the lateral and third ventricles in white light mode (fig 1A). The fiberscope is equipped with dual observation modes for both white light and fluorescence. It was originally developed for photodynamic diagnosis of epithelial dysplasia of the bronchus under excitation with blue light (D-Light AF system; Karl Storz Endoscopy). ${ }^{2}$ A solution of $500 \mathrm{mg}$ of fluorescein sodium was administered intravenously to the patient while the interior of the ventricle was observed with the fiberscope in fluorescence mode. About 20 seconds after the injection, the choroid plexus began to glow yellow-green under the effect of the fluorescein (fig 1B). In the third ventricle the median eminence on the floor and the organum vasculosum of the lamina terminalis also became visible, glowing with yellow-green fluorescence (fig 2).

Fluorescein sodium is commonly used in fluorescein angiography for diabetic retinopathy. The retinal lesions characterised by the damaged blood-retinal barrier can be diagnosed by leakage of the dye. ${ }^{3}$ This same effect can occur in the brain; in most parts of the brain the dye can not pass across the capillary walls because of the blood-brain barrier.
However, several locations in the brain without the blood-brain barrier, such as the circumventricular organs and the choroid plexus, allow the dye to permeate and become visible by fluorescence. Although we could not confirm this phenomenon in the circumventricular organs other than at the median eminence and the organum vasculosm because of limited accessibility with the fiberscope, we think this method of observation may contribute to a better understanding of the circumventricular organs in living humans.

S Kubo, T Inui, K Yamazato Department of Neurosurgery, Tominaga Hospital, Osaka, Japan

Correspondence to: Dr S Kubo, Department of Neurosurgery, Tominaga Hospital, 1-4-48 Naniwa-ku, Osaka 556-0017, Japan; sig-kubo@momo.so-net.ne.jp

\section{References}

1 Ganong WF. Circumventricular organs: definition and role in the regulation of endocrine and autonomic function. Clin Exp Pharmacol Physiol 2000;27:422-7.

2 Malzahn K, Dreyer T, Glanz H, et al. Autofluorescence endoscopy in the diagnosis of early laryngeal cancer and its precursor lesions. Laryngoscope 2002;112:488-93.

3 Richard G. Fluorescein angiography: textbook and atlas. Stuttgart: Georg Thieme Verlag, 1990.
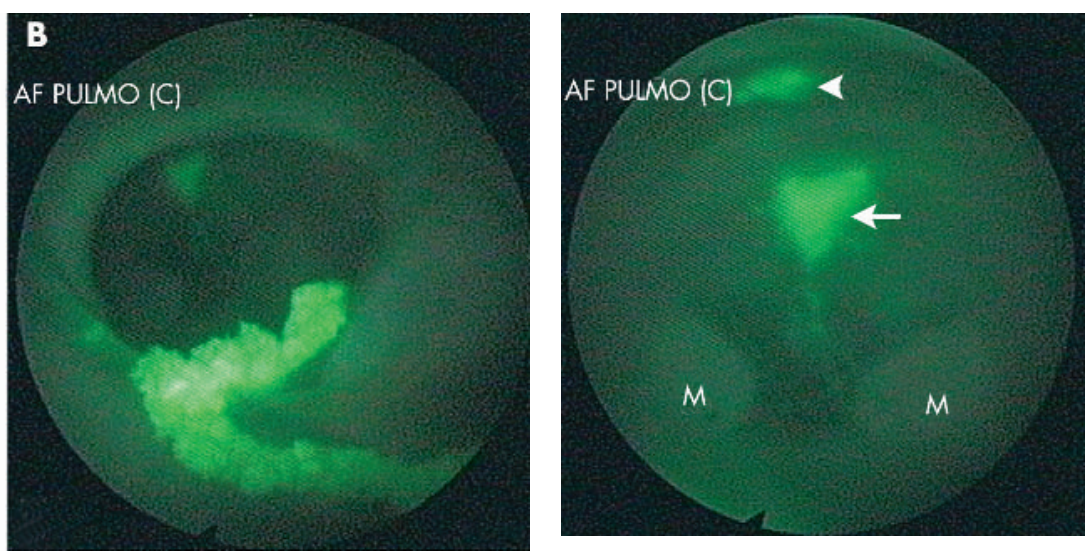

Figure 2 In the third ventricle, the median eminence (arrow) and the organum vasculosum of the lamina terminalis (arrowhead) can be recognised by the fluorescence. $M$, mammillary body. 